\title{
AN EXPLICIT TIME SCHEME WITH LOCAL TIME STEPPING FOR ONE-DIMENSIONAL WAVE AND IMPACT PROBLEMS IN LAYERED AND FUNCTIONALLY GRADED MATERIALS
}

\author{
Radek Kolman ${ }^{1}$, Sang Soon Cho ${ }^{2}$, K.C. Park ${ }^{3}$, and Jose G. Gonzalez ${ }^{4}$ \\ ${ }^{1}$ Institute of Thermomechanics, The Czech Academy of Sciences \\ Dolejkova 5, 18200 Prague, Czech Republic \\ e-mail: kolman@it.cas.cz \\ ${ }^{2}$ Reactor Mechanical Engineering Division, Korea Atomic Energy Research Institute \\ 999-111 Daedeok-Daero, Yuseong-gu, Daejeon 305-353, Korea \\ e-mail: sscho96@kaist.ac.kr \\ ${ }^{3}$ Department of Aerospace Engineering Sciences, University of Colorado at Boulder \\ CO 80309-429, USA \\ e-mail: kcpark@colorado.edu \\ ${ }^{4}$ Escuela Tcnica Superior de Ingeniera, Universidad de Sevilla \\ Camino de los Descubrimientos s/n, Sevilla 41092, Spain \\ e-mail: japerez@us.es
}

Keywords: Wave propagation, Heterogeneous and graded materials, Explicit time integration, Finite element method, Local time stepping, Spurious oscillations.

\begin{abstract}
The standard explicit time scheme (e.g. the central difference method) in finite element analysis is not able to keep accuracy of stress distribution through meshes with different local Courant numbers for each finite element. Therefore in this paper, we suggest and test a two-time step explicit scheme with local time stepping for direct time integration in finite element analysis of wave propagation in heterogeneous solids. The nominated two-time step scheme with the diagonal mass matrix is based on the modification of the central difference method with pullback interpolation and local time stepping. It means that we integrate stress situation on each finite element with local stable time step size. With local time stepping, it is possible to track more accurately a movement of wavefronts for finite element meshes with different local Courant numbers. We present numerical examples of one-dimensional wave propagation in layered and graded elastic bars under shock loading. Based on numerical tests, the presented time scheme is able to eliminate spurious oscillations in stress distribution in numerical modelling of shock wave propagation in heterogeneous materials.
\end{abstract}




\section{INTRODUCTION}

Nowadays, in industrial and engineering applications, number of usages of functionally graded materials (FGM) and materials made of additive manufacturing technology grows up. The reason is that manufacturing processes and tools are available and financially attractive for a wider range of users. Generally, functionally graded materials and materials of light-weight optimized structures offer many advantages in real problems in comparison with conventional materials [1].

In this paper, we focus only on numerical solution of wave propagation in an elastic heterogeneous bar and on accuracy analysis of a nominated explicit scheme in finite element analysis. Wave propagation in functionally graded materials has been analyzed in [2]. Advanced modelling technique of such heterogeneous materials based on the finite volume method has been published in [3]. As we know, the standard explicit time scheme (e.g. the central difference method) in finite element analysis is not able to keep accuracy of stress distribution through meshes with different local Courant numbers for each finite element [4]. The reason is that the smallest local time step size dictates the stability limit and at the place with substantially different time step sizes, the wavefront movement is polluted by dispersion errors of the finite element method (FEM) [5]. Therefore, it is necessary to develop a scheme respecting different wave speeds, mesh sizes and local critical time step sizes. This phenomenon means a big trouble for numerical methods, because wave speed affects stability limit for explicit schemes. Several numerical approaches for elimination of spurious oscillations in heterogeneous media have been developed, as the Park method $[4,6]$ based on pullback interpolation or the Idesman method based on post-processing filtering [7].

\section{AN EXPLICIT TIME SCHEME WITH LOCAL TIME STEPPING: ONE DIMENSIONAL CASE}

In this Section, we present a time scheme with local time stepping for modelling of onedimensional wave propagation in heterogeneous solids. The finite element method [8] based on the Galerkin approximation method with linear shape functions is used. Further, the global mass matrix of lumped type $\mathbf{M}$ and stiffness matrix $\mathbf{K}$ are defined also with Dirichlet boundary conditions and external loading vector $\mathbf{f}(t)$ for each time $t \in\left[0, T_{\text {end }}\right]$. We use the linear theory of elastodynamics [9], therefore the stiffness and mass matrices do not change during computation process. Elemental quantities are marked by the subscript $e$. The nodal elemental displacement vector for the $e$-th element is marked as $\mathbf{u}_{e}$ against the global nodal displacement vector marked $\mathbf{u}$.

Let's assume that we know displacement, velocity and acceleration nodal vectors at the time $t^{n}: \mathbf{u}^{n}, \mathbf{v}^{n}$, and $\mathbf{a}^{n}$, respectively. We estimate the stable time step size $\Delta t$ as $\Delta t=$ $\alpha_{s} \min \left(h_{e} / c_{e}\right)$, where $\alpha_{s}$ is the safety parameter in the range $(0,9], h_{e}$ is the length of the $e$-th finite element, $c_{e}$ is the wave speed of the $e$-th finite element of bar type. In one-dimensional linear theory of wave propagation in solids, the wave speed at the position $x$ is defined as $c(x)=\sqrt{E(x) / \rho(x)}$, where $E$ is the Young's modulus and $\rho$ marks the mass density.

The nominated numerical method for wave propagation in heterogeneous materials is based on the algorithm presented by Park in $[4,6]$. This scheme has been reformulated into the two-time step scheme in [10]. The used time stepping process is consisted of following two computational steps for the predictor-corrector form for numerically elimination of spurious stress oscillations close to wavefront and dispersive properties of the finite element method [5] as follows: 


\section{STEP 1. Pull-back integration with local stepping:}

1a) Integration by the central difference scheme with the local (elemental) critical time step size $\Delta t_{e}^{c r}$ for each finite element at the time $t^{n+c r}=t^{n}+\Delta t_{e}^{c r}$

$$
\begin{gathered}
\left(\mathbf{u}_{f s}^{n+c r}\right)_{e}=\mathbf{u}_{e}^{n}+\Delta t_{e}^{c r} \mathbf{v}_{e}^{n}+\frac{1}{2}\left(\Delta t_{e}^{c r}\right)^{2} \mathbf{a}_{e}^{n} \\
\left(\mathbf{a}_{f s}^{n+c r}\right)_{e}=\left(\mathbf{M}_{e}\right)^{-1}\left[\mathbf{f}_{e}^{n+c r}-\mathbf{K}_{e}\left(\mathbf{u}_{f s}^{n+c r}\right)_{e}\right]
\end{gathered}
$$

The elemental critical time step size $\Delta t_{e}^{c r}$ is set as $\Delta t_{e}^{c r}=h_{e} / c_{e}$ or $\Delta t_{e}^{c r}=2 / \omega_{\text {max }}^{e}$, where $\omega_{\max }^{e}$ is the maximum eigen-angular velocity for the $e$-th separate finite element.

1b) Pull-back interpolation of local nodal displacement vectors at the time $t^{n+1}=t^{n}+\Delta t$ with $\alpha=\Delta t / \Delta t_{e}^{c r}, \quad \beta_{1}(\alpha)=\frac{1}{6} \alpha\left(1+3 \alpha-\alpha^{2}\right), \quad \beta_{2}(\alpha)=\frac{1}{6} \alpha\left(\alpha^{2}-1\right)$

$$
\left(\mathbf{u}_{f s}^{n+1}\right)_{e}=\mathbf{u}_{e}^{n}+\Delta t_{e}^{c r} \mathbf{v}_{e}^{n}+\left(\Delta t_{e}^{c r}\right)^{2} \beta_{1} \mathbf{a}_{e}^{n}+\left(\Delta t_{e}^{c r}\right)^{2} \beta_{2}\left(\mathbf{a}_{f s}^{n+c r}\right)_{e}
$$

1c) Assembling of local contributions of displacement vector from Step 1b.

$$
\mathbf{u}_{f s}^{n+1}=\mathbf{L}\left(\mathbf{u}_{f s}^{n+1}\right)_{e}
$$

where $\mathbf{L}$ is the assembly Boolean matrix.

\section{STEP 2. Push-forward integration with averaging:}

2a) Push-forward predictor of displacement vector at the time $t^{n+1}=t^{n}+\Delta t$ by the central difference scheme with the time step size $\Delta t$.

$$
\mathbf{u}_{c d}^{n+1}=\mathbf{u}^{n}+\Delta t \mathbf{v}^{n}+\frac{1}{2} \Delta t^{2} \mathbf{a}^{n}
$$

2b) Averaging of the total displacement vectors at the time $t^{n+1}=t^{n}+\Delta t$ form Steps 1c and 2 a for given $\theta=[0,1]$.

$$
\mathbf{u}^{n+1}=\theta \mathbf{u}_{f s}^{n+1}+(1-\theta) \mathbf{u}_{c d}^{n+1}
$$

2c) Evaluation of acceleration and velocity nodal vectors at the time $t^{n+1}=t^{n}+\Delta t$.

$$
\begin{gathered}
\mathbf{a}^{n+1}=(\mathbf{M})^{-1}\left[\mathbf{f}\left(t^{n+1}\right)-\mathbf{K} \mathbf{u}^{n+1}\right] \\
\mathbf{v}^{n+1}=\mathbf{v}^{n}+\frac{1}{2}\left(\mathbf{a}^{n}+\mathbf{a}^{n+1}\right)
\end{gathered}
$$

In the following text, we use the averaging parameter as $\theta=0.5$.

\section{NUMERICAL TESTS}

We present results of two numerical tests: elastic wave propagation in layered and graded bars under shock loading. Stress distributions are obtained by the presented explicit scheme with and without local stepping based on the Park method, and they are compared with results given by the central difference method. 


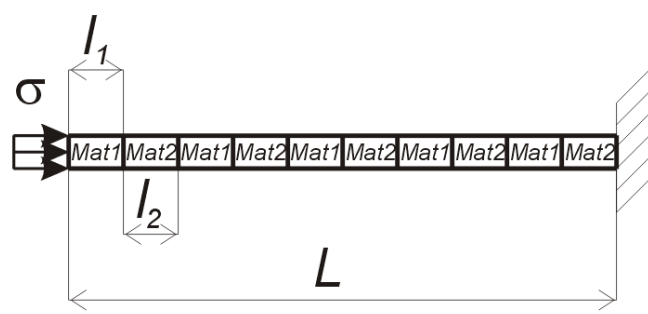

Mat1: $E_{1}, \rho_{1}, C_{1}, Z_{1}$
Mat2: $E_{2}, \rho_{2}, C_{2}, Z_{2}$

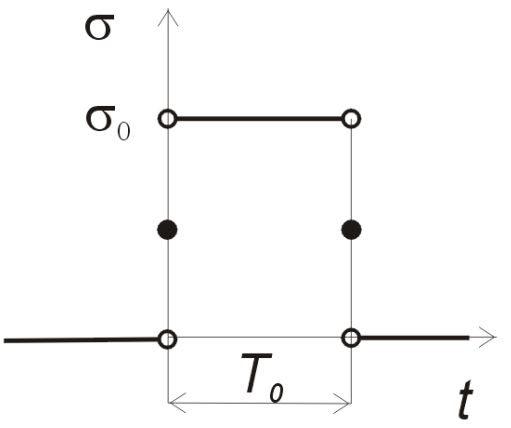

Figure 1: A scheme of a free-fixed layered elastic bar under shock loading.

\subsection{Wave propagation in layered bar}

In this Section, results of numerical solution of wave propagation problem in a layered elastic bar, see Fig. 1, are shown. The bar is consisted of twelve layers with the same lengths, which occur in turn repeatedly as depicted in Fig. 1.

Geometrical and material parameters of the task are set as: the domain length $L=2 \mathrm{~m}$, the cross-section $A=1 \mathrm{~m}^{2}$, Young's modulus corresponding to Material 1 and Material 2 are as $E_{1}=16 \mathrm{~Pa}, E_{2}=1 \mathrm{~Pa}$, the mass density $\rho_{1}=\rho_{2}=1 \mathrm{~kg} / \mathrm{m}^{3}$ and the amplitude of impact pressure $\sigma_{0}=1 \mathrm{~Pa}$, thus the applied force is $F=1 \mathrm{~N}$. We use computational mesh with $N N E=240$ uniform finite elements with length $h=L / N N E=0.008333 \mathrm{~m}$. Time duration of the loading is takes as $T_{0}=0.25 \mathrm{~s}$ and final time of computations is set as $T_{\text {end }}=$ $1.2 \mathrm{~s}$. The critical time step sizes for finite elements of Material 1 and Material 2 are given as $\Delta t_{c r}^{M A T_{1}}=h / c_{1} \approx 0.0021 \mathrm{~s}$ and $\Delta t_{c r}^{M A T_{2}}=h / c_{2} \approx 0.0083 \mathrm{~s}$, respectively. In that case, the value $\Delta t_{c r}^{M A T_{1}}$ dictates the global stability limit so we set the time step size for both tests as $\Delta t=0.5 \Delta t_{c r}^{M A T_{1}} \approx 0.0010 \mathrm{~s}$.

In Fig. 2, the stress distributions for wave propagation in the layered bar at the time $t=$ $1.2 \mathrm{~s}$ are shown. Here one can see results given by the central difference method, the Park method with and without local time stepping. The results are computed for $\Delta t=0.5 \Delta t^{c r}$, where $\Delta t^{c r}$ is the global critical time step size. Based on comparison of the stress distributions along the bar, we can say that the local stepping in direct time integration is able to eliminated spurious oscillations, which are evident in stress distributions given by the central difference method and the original Park method without local time stepping. The cardinal improvement and elimination of stress spurious oscillations in the bar are observed in parts with higher wave speeds, because in that place, the wavefront movement is integrated with smaller time step size and the results are polluted by dispersion errors.

\subsection{Wave propagation in graded bar with linear distribution of elastic modulus}

In this Section, results of numerical solution of wave propagation problem in a graded elastic bar, see Fig. 3, are presented. The elastic modulus of a bar is linearly distributed along the bar, where $E_{1}=16 \mathrm{~Pa}$ and $E_{2}=1 \mathrm{~Pa}$ and the total computational time was set as $T_{\text {end }}=0.75 \mathrm{~s}$. The rest of parameters of the test are the same as in the previous test for a layered bar.

In this test, also the presented explicit scheme with local time stepping produces results without spurious oscillations, only but only small cusps on the corners of stress discontinuities can be observed, see Fig. 4. Further, the improvement of stress spurious oscillations is evident 


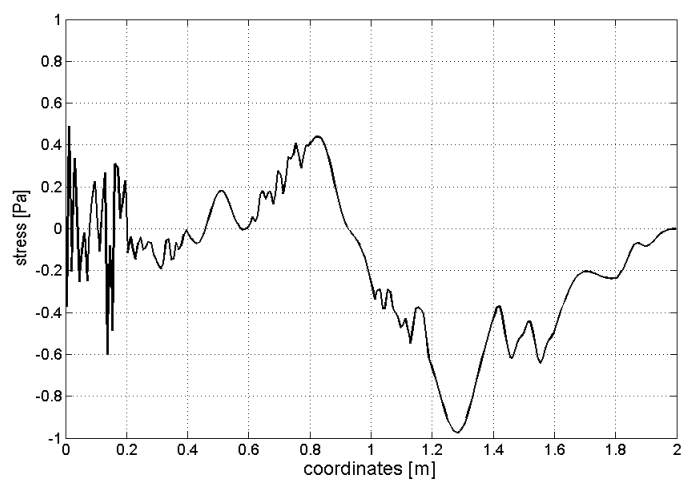

(a)

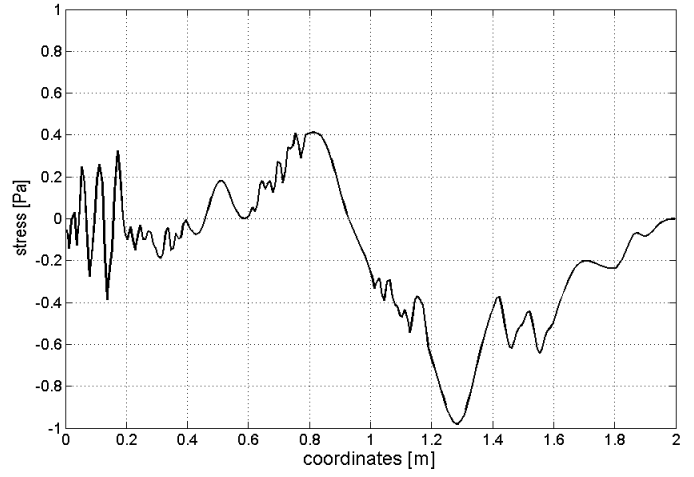

(b)

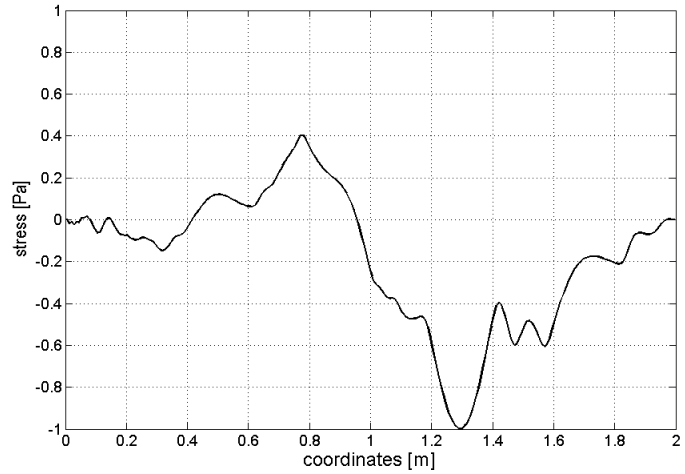

(c)

Figure 2: Stress distributions in a layered elastic bar under shock loading obtained by a) the central difference method, b) the Park method without local stepping, c) the Park method with local stepping.

with comparison of the scheme with and without local stepping, because the nominated local stepping process respects local critical time step size at each material point.

\section{CONCLUSIONS}

In this paper, we have tested the two-time step explicit scheme based on pullback interpolation with local stepping for accurate tracking of elastic waves in heterogeneous media. The layered and graded bar tests have showed accuracy of the presented scheme. The scheme is able to eliminate spurious oscillations in numerical modelling of wave propagation problems against explicit time integration schemes without local time stepping. In the future, we will focus on extension the presented scheme for wave modelling in multidimensional domains.

\section{ACKNOWLEDGEMENT}

The work was supported by the European Regional Development Fund under Grant No. CZ.02.1.01/0.0/0.0/15_003/0000493 (Centre of Excellence for Nonlinear Dynamic Behaviour of Advanced Materials in Engineering), the bilateral project No. ETA-15-03 and the grant projects No. 16-03823S, 17-12925S and 17-22615S of the Czech Science Foundation within institutional support RVO:61388998. 

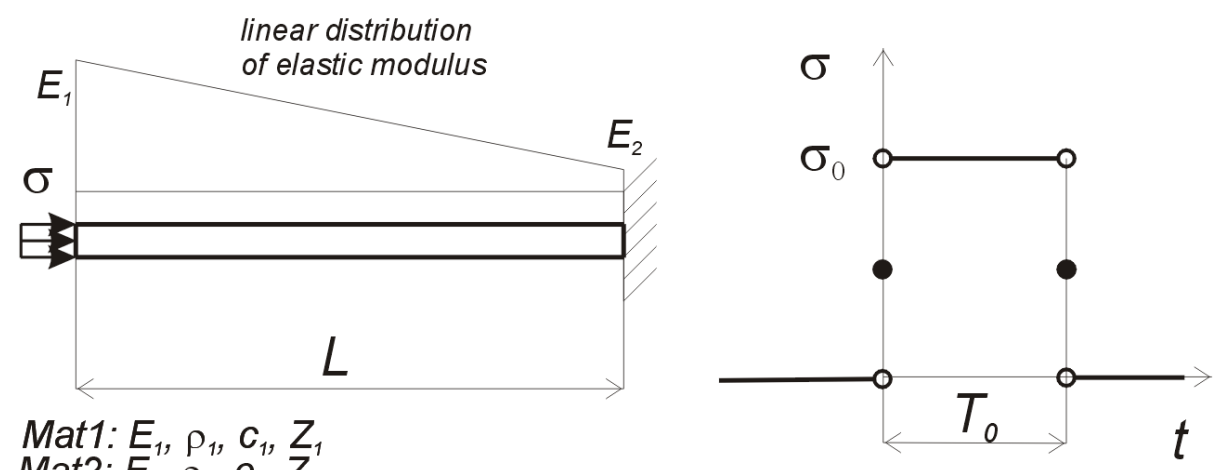

Mat1: $E_{1}, \rho_{1}, c_{1}, Z$

Mat2: $E_{2}, \rho_{2}, C_{2}, Z_{2}$

Figure 3: A scheme of a free-fixed graded elastic bar under shock loading.

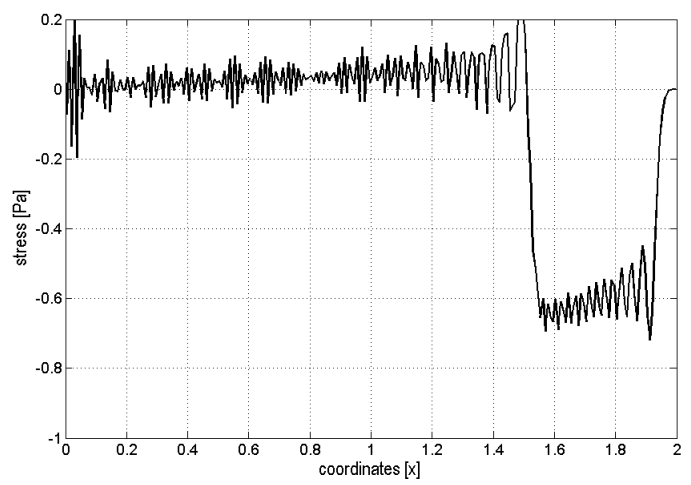

(a)

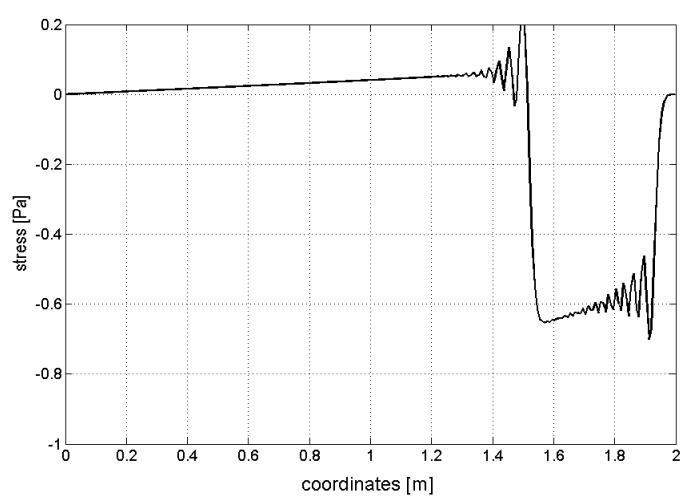

(b)

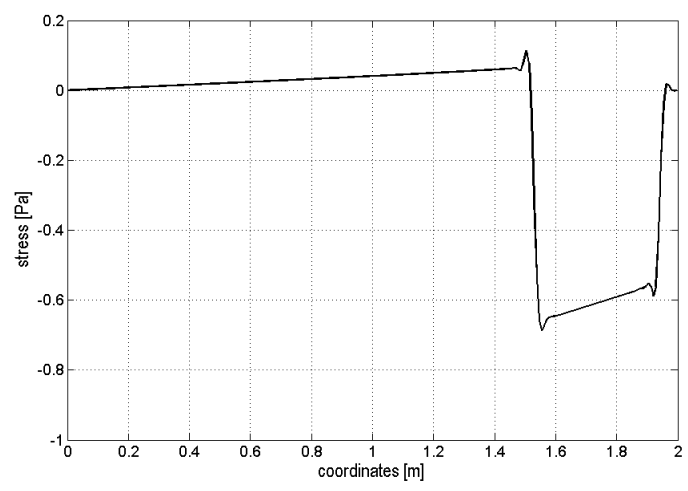

(c)

Figure 4: Stress distributions in a graded elastic bar under shock loading obtained by a) the central difference method, b) the Park method without local stepping, c) the Park method with local stepping.

\section{REFERENCES}

[1] F. Ebrahimi, Advances in Functionally Graded Materials and Structures. InTech, 2016.

[2] T.-C. Chiu, F. Erdogan, One-dimensional wave propagation in a functionally graded elastic medium. Journal of Sound and Vibration, 222, 453-487, 1999. 
[3] A. Berezovski, J. Engelbrecht, G.A. Maugin, Numerical Simulation of Waves and Fronts in Inhomogeneous Solids, World Scientific Pub Co Inc., 2008.

[4] K.C. Park, S.J. Lim, H. Huh, A method for computation of discontinuous wave propagation in heterogeneous solids: basic algorithm description and application to onedimensional problems. International Journal of Numerical Methods and Engineering, 91(6), 622-643, 2012.

[5] R. Kolman, J. Plešek, J. Červ, M. Okrouhlík, P. Pařík, Temporal-spatial dispersion and stability analysis of finite element method in explicit elastodynamics. International Journal for Numerical Methods in Engineering, 106(2), 113-128, 2016.

[6] S.S. Cho, K.C. Park, H. Huh, A method for multi-dimensional wave propagation analysis via component-wise partition of longitudinal and shear waves. International Journal for Numerical Methods in Engineering 95(3), 212-237, 2013.

[7] A. Idesman, Accurate finite-element modeling of wave propagation in composite and functionally graded materials. Composite Structures, 117, 298-308, 2104.

[8] T.J.R. Hughes, The Finite Element Method: Linear and Dynamic Finite Element Analysis. Dover Publications: New York, 2000.

[9] K.F. Graff, Wave motion in elastic solids, first ed.. Oxford University Press, 1975.

[10] R. Kolman, S.S. Cho, K.C. Park, Efficient implementation of an explicit partitioned shear and longitudinal wave propagation algorithm. International Journal for Numerical Methods in Engineering, 107(7), 543-579, 2016. 